\title{
On free differentials on associative algebras *
}

\author{
A. Borowiec ${ }^{\dagger}$ \\ Institute of Theoretical Physics \\ University of Wrocław, Poland, borowiec@plwruw11.bitnet \\ V. K. Kharchenko $\ddagger$ \\ Institute of Mathematics \\ Novosibirsk, Russia, kharchen@math.nsk.su \\ Z. Oziewicz ${ }^{\S}$ \\ Universidad Nacional Autonoma de México \\ México ,oziewicz@redvax1.dgsca.unam.mx
}

November 93, ITP UWr 861/93

\begin{abstract}
A free differential for an arbitrary associative algebra is defined as a differential with a uniqueness property. The existence problem for such a differential is posed. The notion of optimal calculi for given commutation rules is introduced and an explicit construction of it for a homogenous case is provided. Some examples are presented.
\end{abstract}

\section{Introduction}

A differential $d: R \rightarrow{ }_{R} M_{R}$ is called free if the differential of any element $v$ has a unique presentation of the form $d v=d x^{i} \cdot v_{i}$, where $x^{1}, \ldots, x^{n}$ are generators of the algebra and $d x^{1}, \ldots, d x^{n}$ their differentials. Any free differential defines a commutation formula $v d x^{i}=d x^{k} \cdot A(v)_{k}^{i}$, where $A$ : $v \mapsto A(v)_{k}^{i}$ is an algebra homomorphism $A: R \rightarrow R_{n \times n}$. It is easy to see

\footnotetext{
${ }^{*}$ Talk presented by V. Kharchenko at the Third International Conference in Non Associative Algebras and Its Applications, Oviedo, Spain, July 1993

${ }^{\dagger}$ Supported by the Polish Committee for Scientific Research No 224199203

${ }^{\ddagger}$ Supported by the Russian Fund of Fundamental Research No 93-011-16171

$\S$ On leave from the Institute of Theoretical Physics University of Wrocław
} 
that for any homomorphism $R \rightarrow R_{n \times n}$ there exists not more than one free differential. We are going to consider the existence problem of such a differential. We will show that for a given commutation rule $v d x^{i}=$ $d x^{k} \cdot A(v)_{k}^{i}$ a free algebra generated by the variables $x^{1}, \ldots, x^{n}$ has a related free differential. We will define an optimal algebra with respect to a fixed commutation rule. In the homogeneous case this algebra is characterized as the unique algebra which has no nonzero $A$-invariant subspaces with zero differentials. Finally, we will consider a number of example of optimal algebras for different commutation rules. In particular, we will desc two variable commutation rules which define commutative optimal algebra.

This article is closely related to the known Wess and Zumino paper [1]. In our terms, they prove in particular that a system on $n^{2}$ quadratic forms vanish in the optimal algebra if the Yang-Baxter equation holds.

\section{Free differential calculi}

Recall that a differential is a linear mapping from an algebra $R$ to a bimodule $M$ satisfying the Leibniz rule:

$$
d(u v)=d(u) v+u d(v)
$$

Lemma 1.1 $A$ differential d has the uniqueness property iff $\Omega_{d}(R)=R^{b} d(R) R^{b}$ is a free right $R$-module freely generated by $d x^{1}, \ldots, d x^{n}$ (here $R^{b}=R$ if $R$ has a unit and the augmented algebra otherwise).

Due to the lemma the following definition is natural.

Definition 1.2 A differential is said to be free if it has the uniqueness property.

That definition essentially depends on the generating space $V=\sum x^{i} F$. Let us consider as example the case $V=R$. Of course, if $R$ is not finite dimensional, then we have an infinite set of generators. Nevertheless, there exists a free differential with respect to that space of generators. It is exact the universal derivation.

If $d$ is a free differential, then linear maps $D_{k}: R \rightarrow R$ (partial derivatives) can be defined by the formula:

$$
d v=d x^{k} \cdot D_{k}(v)
$$

Those maps satisfy the relations

$$
D_{k}\left(x^{i}\right)=\delta_{k}^{i},
$$


where $\delta_{k}^{i}$ is the Kronecker delta.

Lemma 1.3 A linear map $A_{d}: R \rightarrow R_{n \times n}$ from the algebra $R$ into the algebra of $n$ by $n$ matrices over $R$ given by the formula

$$
A_{d}(v)_{k}^{i}=D_{k}\left(v x^{i}\right)-D_{k}(v) x^{i}
$$

is an algebra homomorphism i.e.

$$
A_{d}(u v)_{k}^{i}=A_{d}(u)_{k}^{l} A_{d}(v)_{l}^{i}
$$

Proof: Let $v \in R$. The left multiplication $A(v): \omega \mapsto v \omega$ is an endomorphism of the right module $\Omega_{d}(R)$. Ring of all endomorphisms of any free module of rank $n$ is isomorphic to the ring of all $n$ by $n$ matrices. Therefore, we can find a homomorphism $A: R \rightarrow R_{n \times n}$ defined by the formulae

$$
v d x^{i}=d x^{k} \cdot A(v)_{k}^{i}
$$

By the Leibnitz rule we have

$$
v d x^{i}=d\left(v x^{i}\right)-d(v) x^{i}=d x^{k}\left[D_{k}\left(v x^{i}\right)-D_{k}(v) x^{i}\right]
$$

therefore,

$$
d x^{k} \cdot A_{d}(v)_{k}^{i}=d x^{k} \cdot A(v)_{k}^{i}
$$

i.e. $A_{d}=A$ and $A_{d}$ is also a homomorphism.

Let us consider a linear map $D: R \rightarrow R^{n}$ of $R$ to the space of columns of height $n$ acted by the formula

$$
D(v)=\left(\begin{array}{c}
D_{1}(v) \\
\vdots \\
D_{n}(v)
\end{array}\right) \text {, i.e. } D=\left(\begin{array}{c}
D_{1} \\
\vdots \\
D_{n}
\end{array}\right)
$$

Proposition 1.4 The map $D$ and homomorphism $A_{d}$ are connected by the relation

$$
D(u v)=D(u) v+A_{d}(u) D(v)
$$

Proof: We have

$$
u d v=u d x^{i} \cdot D_{i}(v)=d x^{k} \cdot A_{d}(u)_{k}^{i} D_{i}(v)
$$


and

$$
d x^{k} \cdot D_{k}(u v)=D_{k}(u) v+A_{d}(u)_{k}^{i} D_{i}(v)
$$

i.e. by the uniqueness condition

$$
D_{k}(u v)=D_{k}(u) v+A_{d}(u)_{k}^{i} D_{i}(v)
$$

The inverse statement is also valid

Proposition 1.5 Let $R$ be an algebra generated by elements $x^{1}, \ldots, x^{n}$ and $A: R \rightarrow R_{n \times n}$ be an algebra homomorphism. If $D: R \rightarrow R^{n}$ is a linear map such that

$$
\begin{gathered}
D_{k}\left(x^{i}\right)=\delta_{k}^{i} \\
D(u v)=D(u) v+A(u) D(v),
\end{gathered}
$$

then the map $\Delta: v \mapsto d x^{k} \cdot D_{k}(v)$ is a free differential, where $\Omega_{\Delta}(R)=$ $\sum d x^{i} \cdot R$ is a free right module with the left module structure defined by commutation rule, i.e. $A_{\Delta}=A$.

Proof: We have to prove $\Delta\left(x^{i}\right)=d x^{i}$ and the Leibnitz formulae. First equality follows from (6) and definition of $\Delta$. Finally

$$
\Delta(u v)=d x^{k} \cdot D(u v)=d x^{k} \cdot\left[D_{k}(u) v+A_{d}(u)_{k}^{i} D_{i}(v)\right]=\Delta(u) v+u \Delta(v) .
$$

A natural question concerning Proposition 1.5 arises here. If a homomorphism $A$ is given, then formula (7) allows one to calculate partial derivatives of a product in terms of its factors. That fact and formula (6) show that for a given $A$ there exists not more then one $D$ satisfying formulas (6) and (7). It is not clear yet whether or not there exists at least one $D$ of such a type. Thus, our first task is to describe these homomorphisms of $A$ for which there exist free differentials with $A_{d}=A$.

Theorem1.6 Let $R=F<x^{1}, \ldots, x^{n}>$ be a free algebra generated by $x^{1}, \ldots, x^{n}$ and $A^{1}, \ldots, A^{n}$ be any set of $n \times n$ matrices over $R$. There exists a unique free differential $d$ such that $A_{d}\left(x^{i}\right)=A^{i}$.

Proof: The map $x^{k} \mapsto A^{k}$ can be uniquely extended to a homomorphism of algebras $A: R \rightarrow R_{n \times n}$. Let us define a map $D$ on monomials in $x^{1}, \ldots, x^{n}$ by induction on its degree. Let $D_{k}\left(x^{i}\right)=\delta_{k}^{i}$ and

$$
D_{k}\left(x^{i} v\right)=\delta_{k}^{i} v+A\left(x^{i}\right)_{k}^{j} D_{j}(v), \quad \text { where } A\left(x^{i}\right)=A^{i}
$$


We have to prove formulae (7) for arbitrary elements $u, v$. It can be done by induction on degree of a monomial $u$.

If this degree is equal to one then (7) implies required result. Let $u=$ $x^{i} u_{1}$. Then by the equality $A(u)=A\left(x^{i} u_{1}\right)=A\left(x^{i}\right) A\left(u_{1}\right)$ and by induction supposition we have

$$
\begin{gathered}
D_{k}(u v)=D_{k}\left(x^{i} u_{1} v\right)=\delta_{k}^{i} u_{1} v+A\left(x^{i}\right)_{k}^{j} D_{j}\left(u_{1} v\right)= \\
=\left[\delta_{k}^{i} u_{1}+A\left(x^{i}\right)_{k}^{j} D_{j}\left(u_{1}\right)\right] v+A\left(x^{i}\right)_{k}^{l} A\left(u_{1}\right)_{l}^{j} D_{j}(v)=D_{k}(u) v+A(u)_{k}^{j} D_{j}(v)
\end{gathered}
$$

Let now $R$ be a non-free algebra defined by the set of generators $x^{1}, \ldots, x^{n}$ and the set of relations $f_{m}\left(x^{1}, \ldots, x^{n}\right)=0, m \in M$ i.e. $R=\hat{R} / \hat{I}$, where $\hat{R}=F<\hat{x}^{1}, \ldots, \hat{x}^{n}>$ is a free algebra and $\hat{I}$ is its ideal generated by elements $f_{m}\left(\hat{x}^{1}, \ldots, \hat{x}^{n}\right), m \in M$.

Let us denote by $\pi$ the natural projection $\hat{R} \rightarrow R$ such that $\pi\left(\hat{x}^{i}\right)=x^{i}$. Since $R_{n \times n}=R \otimes F_{n \times n}, \pi$ defines an epimorphism $\hat{\pi}: \hat{R}_{n \times n} \rightarrow R_{n \times n}$ by the formulae $\hat{\pi}=\pi \otimes i d$, where $i d: F_{n \times n} \rightarrow F_{n \times n}$ is the identity map.

If $A: R \rightarrow R_{n \times n}$ is any homomorphism of algebras, then we have the following diagram of algebra homomorphism

$$
\begin{array}{cccc}
\hat{R} \stackrel{\hat{A}}{\rightarrow} \hat{R} \otimes F_{n \times n} & = & \hat{R}_{n \times n} \\
\pi \downarrow & \downarrow \pi \otimes i d & = & \downarrow \hat{\pi} \\
R \quad \stackrel{A}{\longrightarrow} R \otimes F_{n \times n} & = & F_{n \times n}
\end{array}
$$

Let us choose for any generator $x^{i}$ an arbitrary element $\hat{A}^{i} \in \hat{R}$ such that $\hat{\pi}\left(\hat{A}^{i}\right)=A^{i}$ (recall that $\hat{\pi}$ is epimorphism). Then the map $\hat{x}^{i} \mapsto \hat{A}^{i}$ can be extended to an algebra homomorphism $\hat{A}: \hat{R} \rightarrow \hat{R}_{n \times n}$ (recall that $\hat{x}^{1}, \ldots, \hat{x}^{n}$ are free variables). That homomorphism completes (8) to a commutative diagram. For any relation $f_{m}\left(\hat{x}^{1}, \ldots, \hat{x}^{n}\right)$ we have:

$$
\hat{\pi}\left(\hat{A}\left(f_{m}\left(\hat{x}^{1}, \ldots, \hat{x}^{n}\right)\right)\right)=A\left(\pi\left(f_{m}\left(\hat{x}^{1}, \ldots, \hat{x}^{n}\right)\right)\right)=0 .
$$

Furthermore,

$$
k e r \hat{\pi}=\operatorname{ker}(\pi \otimes i d)=\operatorname{ker} \pi \otimes F_{n \times n}=I_{n \times n},
$$

and finally

$$
\hat{A}\left(f_{m}\left(\hat{x}^{1}, \ldots, \hat{x}^{n}\right)\right) \in \operatorname{ker} \hat{\pi}=I_{n \times n} .
$$


Theorem 1.6 claims that for the homomorphism $\hat{A}: \hat{R} \rightarrow \hat{R}_{n \times n}$ there exi a unique free differential $\hat{d}$ of the free algebra $\hat{R}$.

Definition 1.7 The differential $\hat{d}$ is called a cover differential with respect to the homomorphism $A: R \rightarrow R_{n \times n}$.

Thus we have proved:

Theorem 1.8 For any homomorphism $A: R \rightarrow R_{n \times n}$ there there exists a cover differential $\hat{d}$ of the algebra $\hat{R}$.

Proposition 1.9 An algebra $R$ with generators $x^{1}, \ldots, x^{n}$ and the set of defining relations $\left\{f_{m}, m \in M\right\}$ has a free differential with respect to a homomorphism $A: R \rightarrow R_{n \times n}$ if and only if

$$
\hat{D}_{k}\left(f_{m}\left(\hat{x}^{1}, \ldots, \hat{x}^{n}\right)\right) \in \hat{I}
$$

where $\hat{D}_{k}$ are partial derivatives of the cover differential $\hat{d}$.

Proof: Let the free differential exist. We claim that the diagram

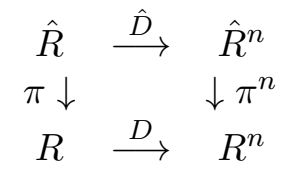

is commutative. Indeed, the difference $\Delta=D \circ \pi-\pi^{n} \circ \hat{D}$ acts trivially on generators:

$$
\Delta_{k}\left(\hat{x}^{i}\right)=D_{k} \pi\left(\hat{x}^{i}\right)-\pi \hat{D}_{k}\left(\hat{x}^{i}\right)=\delta_{k}^{i}-\pi\left(\delta_{k}^{i}\right)=0
$$

Commutativity of (8) implies $A(\pi(f))=\hat{\pi}(A(f))$ and by (7) we have

$$
\begin{gathered}
\Delta(f h)=D(\pi f \cdot \pi h)-\pi^{n} \hat{D}(f h)= \\
=D(\pi f) \pi h+A(\pi f) D(\pi h)-\pi^{n}(\hat{D} \cdot h+\hat{A} f \cdot \hat{D} h)= \\
=\Delta(f) \pi h+A(\pi f) \Delta(h)
\end{gathered}
$$

By evident induction, $\Delta=0$.

Finally for any relation $f_{m}$ we have

$$
\pi \hat{D}\left(f_{m}\left(\hat{x}^{1}, \ldots, \hat{x}^{n}\right)\right)=D\left(\pi\left(f_{m}\left(\hat{x}^{1}, \ldots, \hat{x}^{m}\right)\right)\right)=0
$$

i.e. $\hat{D}_{k}\left(f_{m}\right) \in k e r \pi=\hat{I}$.

Inversely, if $\hat{D}_{k}\left(f_{m}\right) \in \operatorname{ker} \pi=\hat{I}$ then we have

$$
\hat{D}\left(u f_{m} v\right)=\hat{D}(u) f_{m} v+\hat{A}(u) \hat{D}\left(f_{m}\right) v+\hat{A}(u) \hat{A}\left(f_{m}\right) \hat{D}(v)
$$




$$
\equiv \hat{A}(u) \hat{A}\left(f_{m}\right) \hat{D}(v) \quad(\bmod I)
$$

By (8) one has $\hat{\pi} \hat{A}\left(f_{m}\right)=A\left(\pi f_{m}\right)=0$ and $\hat{A}\left(f_{m}\right) \in$ ker $\hat{\pi}=I_{n \times n}$. Therefore $\hat{D}_{k}: \hat{R} \rightarrow \hat{R}$ induce maps $D_{k}: \hat{R} / \hat{I} \rightarrow \hat{R} \stackrel{\pi}{\longrightarrow} R$ in such a way that $D \circ \pi=\pi^{n} \circ \hat{D}$. Finally for arbitrary $u=\pi f \in R$ and $v=\pi h \in R$ we have

$$
\begin{gathered}
D(u v)=D(\pi f \cdot \pi h)=\pi^{n} \hat{D}(f) h+\pi^{n} \hat{A}(f) \hat{D}(h)= \\
=D(\pi f) \pi h+A(\pi f) D(\pi h)=D(u) v+A(u) v
\end{gathered}
$$

and by Proposition 1.5 the proposition is proved.

Corollary 1.10 Let an algebra $R$ be defined by generators $x^{1}, \ldots, x^{n}$ and the set of homogeneous relations $\left\{f_{m}\right\}$ of the same degree. If $A: R \rightarrow R_{n \times n}$ acts linearly on generators $A\left(x^{j}\right)_{k}^{i}=\alpha_{k l}^{i j} x^{l}$, then for the pair $(R, A)$ there exists a free differential iff for all $m$

$$
\hat{d} f_{m}=0
$$

Definition 1.11 An ideal $I$ of a free algebra $\hat{R}=F<\hat{x}^{1}, \ldots, \hat{x}^{n}>$ is said to be comparable with a homomorphism $A: \hat{R} \rightarrow \hat{R}_{n \times n}$ if the factor ring $\hat{R} / I$ has a free differential satisfying the commutation rules

$$
x^{j} d x^{i}=d x^{k} \cdot A\left(x^{j}\right)_{k}^{i} .
$$

If an ideal $I$ is $A$-comparable, then Lemma 1.3 defines a homomorphism $A: r \mapsto A_{k}^{i}(r)$ from the factor algebra into the matrix algebra over it. Thanks to Proposition 1.8, it follows that $I$ is $A$-invariant and $A$-stable in the sense of the following definition:

Definition 1.12 An ideal $J$ of the algebra $\hat{R}$ is said to be $A$-invariant if $A_{k}^{i}(J) \subseteq J$, where $A: r \mapsto A_{k}^{i}(r)$ is a homomorphism. An ideal $I$ is said to be $A$-stable if $D_{k}(I) \subseteq I$ for any of partial derivatives $D_{k}$ defined by a differential $d$ corresponding to $A$ (see Theorem 1.6).

For any homomorphism $A$ there exists the largest $A$-comparable ideal $I(A)$ - the sum of all comparable ideals. It is is again $A$-comparable because a sum of invariant ideals is invariant and a sum of of stable ideals is stable one. 
Now, we are going to describe the ideal $I(A)$ in the homogeneous case. If a homomorphism $A$ preserves a degree, then it must act linearly on generators $A_{k}^{i}\left(\hat{x}^{j}\right)=\alpha_{k l}^{i j} \hat{x}^{l}$. Therefore, the homomorphism $A$ is defined by $\mathrm{t}$ 2-covariant 2-contravariant tensor $A=\alpha_{k l}^{i j}$.

Theorem 1.13 For any 2-covariant 2-contravariant tensor $A=\alpha_{k l}^{i j}$ the ideal $I(A)$ can be constructed by induction as the homogeneous space $I(A)=$ $I_{1}(A)+I_{2}(A)+I_{3}(A)+, \cdots$ in the following way:

1. $I_{1}(A)=0$

2. Assume that $I_{s-1}(A)$ has been defined and $U_{s}$ be a space of all polynomials $m$ of degree $s$ such that $D_{k}(m) \in I_{s-1}(A)$ for all $k .1 \leq k \leq n$. Then $I_{s}(A)$ is the largest $A$-invariant subspace of $U_{s}$.

Proof: First of all, we should note that the maximal $A$-comparable ideal has to be homogenous (graded). It is sufficient to prove that every $A-$ comparable ideal is contained in the homogenous one. Since our free algebra $\hat{R}=\hat{R}_{1}+\hat{R}_{2}+\ldots$ is graded, every element $u \in \hat{R}$ has unique decomposition $u=u_{1}+u_{2}+\ldots$ into homogenous components. Let $J$ be an arbitrary $A$-comparable ideal. Define $J_{s}=\left\{u_{s}: u \in J\right\}$. For $u \in J$ one has $A_{k}^{i}(u)=$ $A_{k}^{i}\left(u_{1}\right)+A_{k}^{i}\left(u_{2}\right)+\ldots \in A_{k}^{i}(J) \subseteq J$ and $\operatorname{deg} A_{k}^{i}\left(u_{s}\right)=\operatorname{deg} u_{s}=s$. Therefore $A_{k}^{i}\left(J_{s}\right) \subseteq J_{s}$. Analogously, $D_{k}(u)=D_{k}\left(u_{1}\right)+D_{k}\left(u_{2}\right)+\ldots \in D_{k}(J) \subseteq J$ and $\operatorname{deg} D_{k}\left(u_{s}\right)=s-1$. So $D_{k}\left(J_{s}\right) \subseteq J_{s-1}$ and the sum $J_{1}+J_{2}+\ldots$ is an $A$-comparable subset. Similarly, $\hat{R}_{t} J_{s} \hat{R}_{p} \subseteq J_{t+s+p}$, hence $J_{1}+J_{2}+\ldots$ is an ideal in $\hat{R}$.

Next step is to prove that $I(A)$ is an ideal. It is sufficient to show that $I_{s-1} \hat{x}^{i}+\hat{x}^{j} I_{s-1} \subseteq I_{s} \forall i, j$. Let $V$ be the space generated by the variables $\hat{x}^{1}, \ldots, \hat{x}^{n}$. Let us prove by induction that $I_{s-1} V+V I_{s-1} \subseteq I_{s}$. We have

$$
\begin{gathered}
D_{k}\left(I_{s-1} V+V I_{s-1}\right) \subseteq D_{k}\left(I_{s-1}\right) V+A_{k}^{j}\left(I_{s-1}\right) D_{j}(V)+D_{k}(V) I_{s-1}+ \\
\quad+A_{k}^{j}(V) D_{j}\left(I_{s-1}\right) \subseteq I_{s-2} \cdot V+I_{s-1}+I_{s-1}+V \cdot I_{s-2} \subset I_{s-1}
\end{gathered}
$$

It follows that $I_{s-1} V+V I_{s-1} \subseteq U_{s}$. Finally, the space $I_{s-1} V+V I_{s-1}$ is $A_{k}^{j}-$ invariant as so are $I_{s-1}$ and $V$. Therefore $I_{s-1} V+V I_{s-1} \subset I_{s}$ and $I$ is an ideal.

Let now $J=J_{1}+J_{2} \ldots$ be an arbitrary (graded) $A$-comparable ideal. We are going to prove by induction that $J_{s} \subseteq I_{s}$. Let $u=\beta_{k} \hat{x}^{k} \in J_{1}$. Then $\beta_{k} x^{k}=0$ in factor-ring $\hat{R} / J$. Therefore $\beta_{k} d x^{k}=0$ and $\beta_{k}=0$ by uniqueness condition. So $u=0$ in the free algebra and $0=J_{1}=I_{1}$. 
Let $J_{s-1} \subseteq I_{s-1}$. By the Proposition 1.9 one has $D_{k}\left(J_{s}\right) \subset J$. All elements from $D_{k}\left(J_{s}\right)$ have degree equal to $s-1$. Therefore $D_{k}\left(J_{s}\right) \subseteq J_{s-1} \subseteq I_{s-1}$ and by the definition of $U_{s}$ we have $J_{s} \subseteq U_{s}$. Finally $J_{s}$ is $A$-invariant space and by the definition of the space $I_{s}$ we obtained $J_{s} \subseteq I_{s}$.

Let us denote by $\hat{R}_{A}$ the factor algebra $\hat{R} / I(A)$. In some sense $\hat{R}_{A}$ is an optimal algebra which has a free differential with respect to the commutation rule $A$. Indeed, Theorem 1.13 shows in particular that if a homogeneous element is such that all elements of the invariant subspace generated by it have all partial derivatives equal to zero, then that element vanishes in the optimal algebra.

We also have proved that there exists maximal algebra which has a free differential with any given commutation rule (this is the free algebra, see Theorem 1.6). Of course, it is very interesting to consider a number of concrete commutation rules $A$ and related algebras $\hat{R}_{A}$.

Example 1. Let us consider the diagonal commutation rule: $x^{j} d x^{i}=$ $d x^{i} \cdot q^{i j} x^{j}$, with the symmetry condition $q^{i j} q^{j i}=1, i \neq j$. If none of the coefficients $q^{i j}$ is a root of a polynomial of the type $\lambda^{[m]} \doteq \lambda^{m-1}+\lambda^{m-2}+\cdots+1$, then the optimal algebra $\hat{R}_{A}$ is equal to $F<x^{1}, \ldots, x^{n}>/\left\{q^{i j} x^{i} x^{j}=\right.$ $\left.x^{j} x^{i}, i<j\right\}$.

If $\left(q^{i i}\right)^{\left[m_{i}\right]}=0,1 \leq i \leq s$ with minimal $m_{i}$ then

$\hat{R}_{A}=F<x^{1}, \ldots, x^{n}>/\left\{q^{i j} x^{i} x^{j}=x^{j} x^{i}, i<j,\left(x^{i}\right)^{m_{i}}=0,1 \leq i \leq s\right\}$.

Example 2. Let $A=0$ i.e. $x^{i} d x^{j}=0$. Then $\hat{d}$ is a homomorphism of right modules and the optimal algebra is free $\hat{R}_{A}=\hat{R}$.

Example 3. Let $x^{i} d x^{j}=-d x^{i} \cdot x^{j}$. Then the optimal algebra is the smallest possible algebra generated by the space $V$ i.e. $\hat{R}_{A}=F<x^{1}, \ldots, x^{n}>$ $/\left\{x^{i} x^{j}=0\right\}$.

Example 4. Let $x^{1} d x^{1}=d x^{1} \cdot\left(\alpha_{2} x^{2}+\cdots+\alpha_{n} x^{n}\right)$ and $x^{i} d x^{j}=-d x^{i} \cdot x^{j}$ if $i \neq 1$ or $j \neq 1$. Then the optimal algebra is almost isomorphic to the ring of polynomials in one variable. More precisely, $\hat{R}_{A}=F\left\langle x^{1}, \ldots, x^{n}\right\rangle$ $/\left\{x^{i} x^{j}=0\right.$, unless $\left.i=j=1\right\}$.

Example 5. If $n=2$ and $x^{1} d x^{1}=d x^{1} \cdot \mu x^{2}, x^{1} d x^{2}=-d x^{1} \cdot x^{2}, x^{2} d x^{1}=$ $-d x^{2} x^{1}, \quad x^{2} d x^{2}=d x^{2} \cdot \lambda x^{1}$, then the optimal algebra is isomorphic to the direct sum of two copies of the polynomial algebra $\hat{R}_{A}=F<x^{1}, x^{2}>$ $/\left\{x^{1} x^{2}=x^{2} x^{1}=0\right\}$. 
Finally, we can formulate result which describes numbers of commutation rules in two variables for which the optimal algebra is commutative.

Theorem 1.14 In the two variable case, the following five series have commutative optimal algebra:

$$
\begin{aligned}
& \text { 1. } x^{1} d x^{1}=d x^{1} \cdot u+d x^{2} \cdot s, \quad x^{1} d x^{2}=d x^{1} \cdot w+d x^{2} \cdot\left(\lambda s+x^{1}\right) \text {, } \\
& x^{2} d x^{1}=d x^{1} \cdot\left(w+x^{2}\right)+d x^{2} \cdot(\lambda s), \\
& x^{2} d x^{2}=d x^{1} \cdot(\lambda w)+d x^{2} \cdot\left(\lambda^{2} s-\lambda u+w+\lambda x^{1}+x^{2}\right) ; \\
& \text { 2. } x^{1} d x^{1}=d x^{1} \cdot\left(x^{1}+\lambda w+s\right)+d x^{2} \cdot w, \quad x^{1} d x^{2}=d x^{1} \cdot \gamma w+d x^{2} \cdot\left(x^{1}+s\right) \text {, } \\
& x^{2} d x^{1}=d x^{1} \cdot\left(x^{2}+\gamma w\right)+d x^{2} \cdot s, \quad x^{2} d x^{2}=d x^{1} \cdot \gamma s+d x^{2} \cdot\left(x^{2}+\gamma w-\lambda s\right) ; \\
& \text { 3. } \quad x^{1} d x^{1}=d x^{1} \cdot\left(x^{1}+\gamma w\right), \quad x^{1} d x^{2}=d x^{1} \cdot w+d x^{2} \cdot x^{1}, \\
& x^{2} d x^{1}=d x^{1} \cdot\left(x^{2}+w\right), \quad x^{2} d x^{2}=d x^{1} \cdot s+d x^{2} \cdot\left(x^{2}+w-\gamma s\right) ; \\
& \text { 4. } \quad x^{1} d x^{1}=d x^{1} \cdot u, \quad x^{1} d x^{2}=d x^{2} \cdot x^{1}, \\
& x^{2} d x^{1}=d x^{1} \cdot x^{2}, \quad x^{2} d x^{2}=d x^{2} \cdot v ; \\
& \text { 5. } \quad x^{1} d x^{1}=d x^{1} \cdot u, \quad x^{1} d x^{2}=d x^{2} \cdot u, \\
& x^{2} d x^{1}=d x^{1} \cdot x^{2}+d x^{2} \cdot\left(u-x^{1}\right), \quad x^{2} d x^{2}=d x^{1} \cdot w+d x^{2} \cdot v .
\end{aligned}
$$

where $u, v, w, s$ are arbitrary elements from the space $V$ and $\lambda, \gamma$ are parameters from the base field.

Remark. The above series are not independent. For example, the standard Newton-Leibnitz calculi $\left(x^{i} d x^{j}=d x^{j} \cdot x^{i}\right)$ belongs, as a special case to each of them, by putting $s=w=0, u=x^{1}, v=x^{2}$. More detailed discussion of the above examples and classification theorem for calculi with a commutative optimal algebra will be given elsewhere [2].

\section{References}

[1] J. Wess and B. Zumino, Covariant differential calculus on the quantum hyperplane. Nuclear Physics 18B (1990), p.303 .

[2] A. Borowiec, V. K. Kharchenko and Z. Oziewicz Differentials with Uniqueness Property - in preparation. 